\title{
How will the human genome project change cardiovascular medicine?
}

Cardiovascular disorders represent a major public health concern because of their high prevalence, morbidity, and mortality. Indeed hypertension, coronary artery disease, heart failure, stroke or peripheral vascular diseases are a major contributor to mortality and, because of the increase in life expectancy and aging, a pandemic both in developed and developing countries. The completion of the first working draft of the human DNA sequence, ${ }^{1}$ which was recently published, is considered by the international community as a major scientific contribution of the human genome project (HGP), an unprecedented international collaborative programme. How the HGP will affect cardiovascular medicine is therefore a question of paramount importance because of the socioeconomic impact of these disorders.

\section{Promises}

Human DNA sequencing opens the door wide on promising perspectives in cardiovascular genetics.

NEW MORBID GENES AND VARIANTS

Although positional cloning has allowed major breakthroughs in the understanding of the genetic background of various familial and often rare diseases, including myocardial disorders (hypertrophic cardiomyopathy), arrhythmic disorders (long QT syndrome), vascular diseases (Marfan syndrome) or various congenital heart diseases, a number of monogenic cardiovascular disorders remain unravelled. ${ }^{2}$ Arrhythmogenic right ventricular cardiomyopathy, dilated cardiomyopathy or mitral valve prolapse have been associated with chromosomal loci but the underlying molecular defect remains unknown. The publication of lists of genes included in the morbid loci associated with these disorders should therefore allow a more rapid identification of morbid genes.

An even more exciting impact of the HGP on cardiovascular medicine is the identification of genetic variants associated with the more prevalent complex diseases such as atherosclerosis or hypertension. Until now, the genetic strategies used to identify susceptibility genes in these disorders have been in part limited by the small number of variants available. The systematic description of all common variants contained in the human genome, including single nucleotide polymorphism, ${ }^{3}$ should allow the development of new and powerful strategies to unravel the genetic components of these complex cardiovascular disorders.

GENETIC RISK, "REVERSE MEDICINE", AND PREVENTION The identification of numerous new morbid genes should provide useful diagnostic tools for the assessment of the genetic risk in a given family, especially in the younger generation where penetrance is often incomplete as observed in hypertrophic or dilated cardiomyopathy. Risk stratification based on genotyping is another interesting perspective in cardiology: in hypertrophic cardiomyopathy, mutations occurring in the myosin binding cardiac protein
C gene are associated with a significantly smaller risk of death or disease related events than mutations in the $\beta$ myosin heavy chain gene. ${ }^{4}$ The genetic status has therefore become an element of prognostic evaluation in affected subjects.

Another perspective includes "reverse medicine"-for example, reassessment of the accuracy of the clinical criteria used for phenotyping, or the search for new, more sensitive and specific diagnostic criteria based on the genetic status. ${ }^{5}$ Since phenocopy is a common situation in cardiovascular diseases (for example, hypertrophic cardiomyopathy and hypertensive heart, dilated cardiomyopathy and ischaemic heart disease with cardiac dilation), and since the phenotype appears highly variable among individuals sharing the same mutation, this "cross talk" process between geneticists and cardiologists is important for the purpose of the definition of well characterised phenotypes.

The impact of the HGP on the prevention of complex multifactorial disorders is another potential important contribution to cardiovascular medicine. The identification of various susceptibility genes in combination with behavioural and environment factors in an individual could lead to the development of special prevention programmes or even early medical intervention in order to slow down the development of atherosclerosis or hypertension.

\section{GENE BASED PHARMACOTHERAPY}

Preliminary data suggest that unravelling the molecular defects involved in various monogenic disorders may result in specific therapeutic strategies: sodium channel blockers might bring benefit in long QT syndrome related to mutations in the sodium channel gene but not in the potassium channel gene. ${ }^{6}$ Women bearing the factor V Leiden variant are at high risk of cerebral vein thrombosis when using oral contraceptives. $^{7}$

In common complex disorders, a major challenge will be to identify genotype related differences in drug efficacy or toxicity. Since cardiovascular pharmacology is characterised by polypharmacy and mass treatment, pharmacogenomics is of particular importance to identify subgroups of patients likely to benefit from various medical interventions.

The design of new treatments based on gene products such as rtPA (recombinant tissue plasminogen activator) for acute myocardial infarction, or etanercept (tumour necrosis factor $\alpha(\mathrm{TNF} \alpha)$ soluble receptor fusion protein) for heart failure is another promising perspective, and the identification of new genes with new functions should foster this rapidly expanding area.

\section{Questions and doubts}

A COLOSSAL COMPLEXITY

Two important items of information have been provided by studies conducted in monogenic cardiac disorders over the past decade.

Firstly, genetic heterogeneity is the rule for many disorders, including hypertrophic, dilated cardiomyopathies, and the long QT syndrome. The high number of genes, 
mutations, and chromosomal loci reported so far in different familial entities, the fact that some of these genes are confined to one or a few pedigrees, and finally the fact that many mutations are "private", makes genetic testing a technical challenge which will need the development of powerful automated technologies such as microarrays.

Secondly, establishing the phenotype-genotype relation is a difficult task. Because of the genetic heterogeneity, the role of other factors such as gene-gene interaction or the role of environment, it is presently difficult to establish precise correlations between genotype and prognosis or disease related morbid events in diseases such as hypertrophic cardiomyopathy, and many exceptions to the initial conclusions have been reported making genetic counselling difficult even within one single pedigree. Obviously, there is a need for a worldwide database in order to clarify this issue and we must recognise that in these diseases genetic based risk stratification is at present beyond our possibilities. In complex diseases, the identification of the impact of genetic variants on diagnosis, prognosis or treatment will need a rigorous methodology based on large populations samples, ethnic homogeneity, and replication of results in order to avoid contradictory results as observed with angiotensin converting enzyme I/D polymorphism and the risk of coronary artery disease. ${ }^{8}$ Moreover, in these multifactorial diseases, the magnitude of the risk conferred by a variant is usually low and depends on a complex and unknown interplay with environmental factors or with other genes. In this regard, elucidating the role of modifier genes which influence the clinical presentation or the outcome of otherwise familial or non-familial disorders will be a major scientific challenge. ${ }^{9}$ It will require large, perfectly well characterised phenotyped populations and powerful complex statistical methods to identify an effect which remains of small magnitude and may be the net result of the combination of aggravating and protecting genes, a domain which remains largely unexplored.

\section{KNOWLEDGE GAP}

Thanks to the HGP, a number of new genes encoding proteins with unknown function will be in the public domain. The next step will therefore be to explore the expression and the role of these newly discovered proteins in normal cardiomyocytes, smooth muscle cells, and in various experimental models. The fact that mutations in a similar gene, LMNA, may result in apparently unlinked diseases such as Emery-Dreyfuss muscular dystrophy, cardiomyopathy or lipodystrophy introduces another degree of complexity. ${ }^{10}{ }^{11} \mathrm{~A}$ tremendous scientific effort is therefore needed for integrated cardiovascular physiology and pathophysiology based on these new tools at a time when the academic sector lacks physiologists. It is therefore of paramount importance to return to cellular and organ physiology to take advantage of this wealth of new information and concepts, leading to the so called "functional genomics".

\section{TREATMENT}

Gene based therapy in many of the cardiovascular disorders reviewed here appears uncertain. One reason for this is the high genetic heterogeneity reported in a single disease making the choice of a gene of interest difficult. Another reason is the fact that the molecular mechanism of the mutation is often dominant negative and not haplo-insufficiency. ${ }^{12}$ Finally, in multifactorial disease where the genetic abnormalities play only a minor risk, this strategy might not be fruitful.

PREDICTIVE MEDICINE

The remarkable efforts made to develop predictive medicine or presymptomatic counselling are partly limited by the fact that few treatments are available once the disease appears, as in hypertrophic cardiomyopathy, whereas prophylactic surgery may be advised in other disorders such as Marfan syndrome. The medical benefit brought to the patient by predictive medicine must therefore be balanced by the psychological burden of diagnosis and the therapeutic options available. Obviously, there is a need for a cautious multidisciplinary approach including cardiologists, psychologists, and geneticists.

\section{Conclusion}

Thanks to the HGP, we are facing an unprecedented revolution in concepts of biological pathways and molecular basis of cardiovascular disorders. To allow the translation of this new information from laboratories to the health care setting, it is mandatory to design appropriate educational programmes targeted to physicians and patients. The gap of knowledge is enormous among cardiologists and the public. Fair scientific information pinpointing the perspectives but also the limitations of cardiovascular genetics is the next step in order to address the issues raised by the HGP on confidentiality, ethics, insurability, and genetic discrimination.

Comprehensive education programmes should therefore be the next step in order to ensure proper use of the new information available by the physicians and to sensitise the cardiovascular community to the wealth of scientific, medical, and therapeutic implications brought by the HGP.

\section{MICHEL KOMAJDA} PHILIPPE CHARRON

Association Claude Bernard and UPRES EA 2390 "Genetics of Heart Failure", Hôpital Pitié Salpêtrière,

75013 Paris,

France

michel.komajda@psl.ap-hop-paris.fr

1 International Human Genome Consortium. Initial sequencing and analysis of the human genome. Nature 2001;409:860-921.

2 Roberts R Schwartz K. Myocardial diseases. Circulation 2000;102:IV-34-9.

3 International SNP Map Working Group. A map of the human genome International SNP Map Working Group. A map of the human genome
sequence variation containing 1.42 million single nucleotide polymorsequence variation containing 1.42

phisms. Nature 2001;409:928-33.
4 Charron PH, Dubourg O, Desnos M, et al. Clinical features and prognostic Charron $\mathrm{PH}$, Dubourg $\mathrm{O}$, Desnos $\mathrm{M}$, et al. Clinical features and prognostic
implications of familial hypertrophic cardiomyopathy related to the cardiac myosin binding protein C gene. Circulation 1998;97:2230-6.

5 Charron Ph, Dubourg O, Desnos M, et al. Diagnostic value of electrocardiography and echocardiography for familial hypertrophic cardiomyopathy in a genotyped adult population. Circulation 1997;96:214-19.

6 Schwartz PJ, Priori SG, Locati EH, et al. Long QT syndrome patients with mutations of the SCN5A and HERG genes have differential responses to $\mathrm{Na}+$ channel blockade and to increases in heart rate: implications for genespecific therapy. Circulation 1995;92:3381-6.

7 Martinelli I, Sacchi E, Landi G, et al. High risk of cerebral-vein thrombosis in carriers of a prothrombin-gene mutation and in users of oral contraceptives. N Engl f Med 1998;338:1793-7.

8 Agerholm-Larsen B, Nordestgaard BG, Steffensen R, et al. ACE gene polymorphism: ischaemic heart disease and longevity in 10150 individuals. A case referent and retrospective cohort study based on the Copenhagen A case referent and retrospective cohort study
city heart study. Circulation 1997;95:2358-67.

9 Tesson F, Dufour C, Moolman JC, et al. The influence of the angiotensin-I converting enzyme genotype in familial hypertrophic cardiomyopathy varies with the disease gene mutation. F Mol Cell Cardiol 1997;29:831-8.

10 Bonne G, Di Barletta MR, Varnous S, et al. Mutations in the gene encoding lamin A/C cause autosomic dominant Emery Dreyfuss muscular dystrophy. Nat Genet 1999;21:285-8

11 Shackleton S, Lloyd DJ, Jackson SN, et al. LMNA, encoding lamin A/C, is mutated in partial lipodystrophy. Nature Genet 2000;24:153-6.

12 Bonne G, Carrier L, Richard P,et al. Familial hypertrophic cardiomyopathy: from mutations to functional defects. Circ Res 1998;83:579-93. 